\title{
The NECOS Approach to End-to-End Cloud-Network Slicing as a Service
}

\author{
Stuart Clayman, Augusto V. Neto, Fábio L. Verdi, Sand Correa, Silvio Sampaio, Ilias Sakelariou, \\ Lefteris Mamatas, Rafael Pasquini, Kleber Cardoso, Francesco Tusa, Christian Rothenberg, and Joan Serrat
}

\begin{abstract}
Cloud-network slicing is a promising approach to serve vertical industries delivering their services over multiple administrative and technological domains. However, there are numerous open challenges to provide end-to-end slices due to complex business and engineering requirements from service and resource providers. This article presents a reference architecture for the cloud-network slicing concept and the practical realization of the Slice-as-a-Service paradigm, which are key results from the NECOS (Novel Enablers in Cloud Slicing) project. The NECOS platform has been designed to consider modularity, separation of concerns, and multi-domain dynamic operation as prime attributes. The architecture comprises a set of inter-working components to automatically create, manage, and decommission end-to-end Cloud-Network slice instances in a lightweight manner. NECOS orchestrates slices at run-time, spanning across core/edge data centres and wired/wireless network infrastructures. The novelties of the multi-domain NECOS platform are validated through three proof-of-concept experiments: (i) a touristic content delivery service slice deployment featuring on-demand virtual infrastructure management across three countries in different continents to meet particular slice requirements; (ii) intelligent slice elasticity driven by machinelearning techniques; and (iii) marketplace-based resource discovery capabilities.
\end{abstract}

Index Terms-Slice-as-a-Service, Data Center Slicing, Network Slicing, End-to-End Cloud-Network Slicing

\section{INTRODUCTION}

Modern Internet services are characterized by large-scale deployments of distributed software nodes to serve myriads of service consumers. Increasingly, many of these services need to be adaptable to challenging and stringent application requirements, such as ultra-low delay. Such demanding services must co-exist with "standard" applications, a situation that introduces conflicting requirements over shared infrastructures. The availability of distributed computing, storage, and

Stuart Clayman and Francesco Tusa are with the University College London, London, England: s.clayman@ucl.ac.uk, francesco.tusa@ucl.ac.uk.

Augusto Neto and Silvio Sampaio are with Federal University of Rio Grande do Norte, Natal/RN, Brazil; augusto@dimap.ufrn.br, silvio@imd.ufrn.br.

Fabio Verdi is with Federal University of São Carlos, Sorocaba, Brazil: verdi@ufscar.br.

Sand Correa and Kleber Cardoso are with Federal University of Goiás, Goiânia, Brazil: sand,kleber@inf.ufg.br.

Illias Sakelariou and Eleftherios Mamatas are with the University of Macedonia, Thessaloniki, Greece: iliass@uom.edu.gr, emamatas@uom.edu.gr.

Rafael Pasquini is with Fderal University of Uberlândia, Uberlândia, Brazil: rafael.pasquini@ufu.br.

Christian Rothenberg is with University of Campinas, Campinas, Brazil: chesteve@dca.fee.unicamp.br.

Joan Serrat is with Universitat Politecnica de Catalunya, Barcelon, Spain serrat@tsc.upc.edu.

Manuscript received August 05, 2020; networking resources, along with diverse service requirements and varying workloads, differs from traditional deployments on large-scale central Data Centers (DC).

To embrace new environments of geographically spread cloud capabilities, Multi-Access Edge Computing (MEC), telco clouds, and network slicing are emerging, as network operators and service providers seek to extend their service platform footprints. Network slicing provides the ability to divide a single physical network infrastructure into multiple co-existing and isolated logical networks, with each slice being tailored to cope with and address the networking requirements of a particular service. This logical grouping of networking resources can cover the complete end-to-end path, including both radio/network equipment and physical cloud server resources, and may cross several administrative boundaries.

An evolutionary view of the implementation of network slicing [1] harnesses the network design and operation principles of the combined utilisation of both Network Functions Virtualization (NFV) with Software-Defined Networking (SDN). Surveying the current state of affairs [2], [3] reveals that: (i) there is still little evidence [4] of works that have attempted holistic end-to-end slicing approaches spanning across multiple administrative domains of network and cloud deployments, and (ii) those available are not sufficient to deliver the broadest and most ambitious visions around network slicing.

The NECOS (Novel Enablers in Cloud Slicing) project [5] advances the state-of-the-art by offering Slice-as-a-Service capabilities through a novel modular, multi-domain, end-toend cloud-network slicing architecture with dynamic operation and a separation of concerns in the design. A NECOSprovisioned cloud-network slice instance represents a group of managed elements: a set of infrastructure facilities (network, edge/cloud) plus networking functions from providers; a set of manageable resources (i.e., connectivity, compute, and storage); and the service applications that feature operations and attributes specially devised for the requirements of specific industry verticals. Each activated cloud-network slice instance holds a set of computing and networking resources across a number of domains, whereby tenants can deploy services and/or applications running in a virtualised form. To ensure the appropriate deployment, slices that span across a shared multi-domain infrastructure are orchestrated in a way that matches the dynamic end-to-end quality requirements of the slice, while still being independent of each other. Towards this direction, NECOS introduces the following novel features:

F1. The Slice-as-a-Service model, which fully addresses the lifecycle (creating, reconfiguring, and decommissioning) of cloud-network slice instances on-demand and at run- 
time, allowing end-to-end multi-tenancy highly-isolated service provisioning. The tenant specifies slice requirements through appropriate service descriptors and APIs, such that the NECOS platform creates a new end-toend cloud-network slice instance and associated slice operation-specific management facilities.

F2. The Lightweight Slice Defined Cloud (LSDC) architecture, for tackling the complexity of large-scale virtualized infrastructure environments, and leveraging intelligent management and orchestration functions. Instead of overloading heavyweight MANO systems with extra slicing capabilities, LSDC follows a modular, lightweight, microservices architectural approach.

F3. Multi-domain Cloud-Network Slicing enabling cooperative business models and large-scale slice deployments, over both network and cloud providers.

F4. Intelligent Elasticity capabilities, supporting both horizontal and vertical slice elasticity, harnessing MachineLearning (ML) techniques.

F5. An Infrastructure Manager On-Demand model for allocating dedicated management and control points that gives the ability to allocate a new Virtual Infrastructure Manager (VIM) on-demand for each DC slice [6], and a new Wide-area Infrastructure Manager (WIM) ondemand for each network slice part [7].

F6. A Resource Marketplace performing dynamic discovery of cloud and network resources for slice parts across multiple geographic domains, via an online Resource Marketplace, rather than having predetermined providers sets previously configured in a federation [8].

The main contributions of this article are three-fold: (i) an overview of the capabilities required to support end-to-end cloud-network slicing over multiple cloud and network resource providers; (ii) the final design of the novel NECOS LSDC architecture, offering those capabilities via the Sliceas-a-Service model; and (iii) an evaluation of the NECOS modular architecture and the above six NECOS features through the testbed experimentation of three different scenarios built upon proof-of-concept software prototypes. The work described in [9] introduces the initial proposal in the context of the project, while [10] covers an earlier feasibility study of NECOS. This article extends both previous publications by exercising the architecture and system platform (fully detailed in project deliverables [11], [12]) in three proof-of-concept experiments on the final implementation. Finally, it should be noted that the NECOS platform has been released as opensource artifacts, featuring its novel capabilities through five different demonstrators [5].

\section{RELATED WORK}

In our previous work [3], we surveyed with an architectural perspective work from six standardization bodies (ITU-T, NGMN, IETF, 3GPP, ETSI, and ONF) related to 5G slicing, and from eight relevant project initiatives considered to be the most closely related to NECOS. We identified 14 key capabilities for slicing, including connectivity resource slicing, connectivity service slicing, network cloud slicing, end-to-end

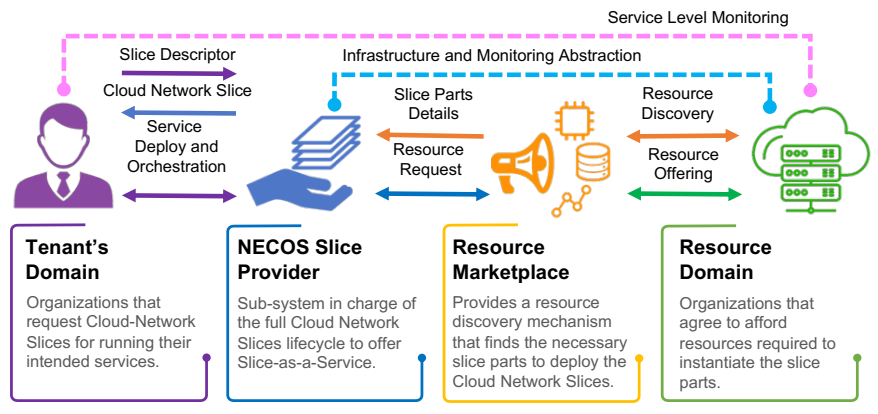

Fig. 1. The four main actors participating in the NECOS architecture.

slicing, multi-domain slicing, uniform lifecycle management, tenant slice management, slices as a service, VIM/WIM ondemand, marketplace for slices, network elasticity, cloud elasticity, and full monitoring. Then, the six standards and eight project initiatives were analyzed from the point of view of the fulfilment of these key capabilities, and, as a result, none of them fulfilled all of the capabilities. Furthermore, we observed that those standards presented divergences in their approach to where slicing was done and what their scope was. Our survey results suggest that those approaches do not fully encompass all of the needs that network slicing could bring about.

Literature surveys on slicing [1], [2] confirm that the concept of slicing is broadening on multiple perspectives and that SDN and NFV are part of the solution [4]. Much of the related work on slicing is limited to the $5 \mathrm{G}$ domain, and only a few consider joint slicing of cloud and network domains or fully address heterogeneous service demands.

Recent efforts at the ETSI Zero-touch network and Service Management Industry Specification Group (ZSM ISG) [13] have resulted in a ZSM framework to deliver fully automated network and service management in multi-domain environments for $5 \mathrm{G}$ and beyond, based on a model-driven architecture. The proposed approach also has an end-to-end slice design, which can be recursively built via combining multiple slice subnets in different segments of the infrastructure. NECOS relies on a similar model, but although not recursive, it also considers the compute slice parts in the possible end-toend slice building blocks. In the NECOS model, compute and network slices can be directly accessed, and securely attached to an end-to-end slice, by the provider that initiated the slice creation workflow. This can overcome the need for peer-topeer Orchestrator interactions after a slice has been put in place, increasing resource isolation and performance.

\section{NECOS Platform Actors And Business CASE}

The main actors and architectural tenets of NECOS are illustrated in Figure 1. Details about each actor, their top-level interactions, and an exemplar business case are presented next.

\section{A. The Tenant}

A NECOS tenant is an organisation requiring cloud-network slices for their own services to run. A tenant will initiate the slice creation process by presenting a request specifying a slice descriptor covering different scopes (e.g., commercial, geographical, contractual commitments). 


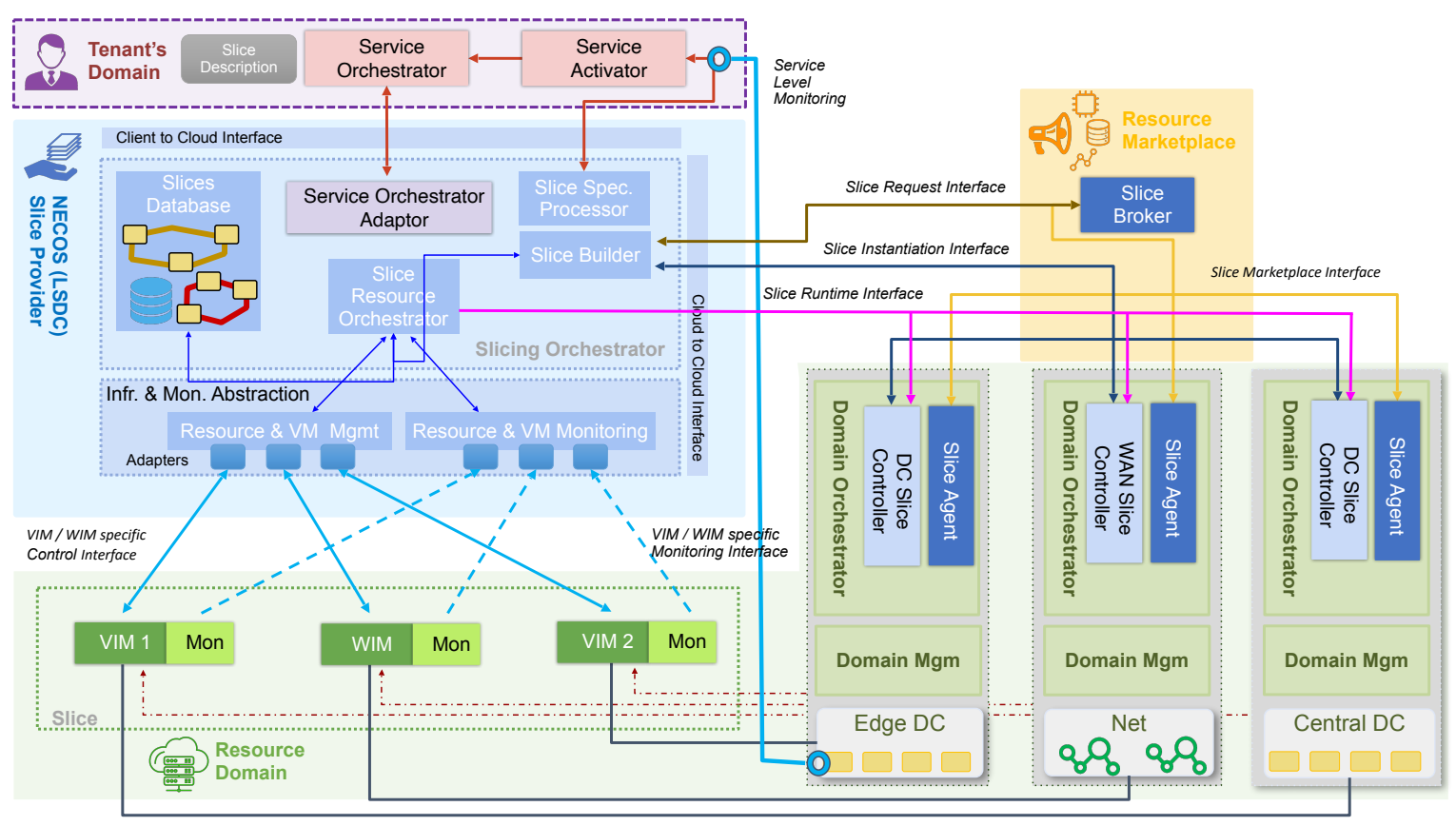

Fig. 2. Functional view of the NECOS architecture.

\section{B. NECOS Slice Provider}

The NECOS slice provider is a management entity responsible for creating end-to-end cloud-network slice instances from a set of constituent network and DC-like slice parts, with each NECOS created slice instance being mapped to a set of federated resources. When a slice is requested, the NECOS slice provider initiates a search via the resource marketplace for slice parts. Upon the receipt of answers from the Marketplace, in the form of alternative resource offers for each part, the slice provider selects and combines the slice parts into a single aggregated slice. The slice provider is in charge of orchestrating end-to-end cloud-network slices, as well as their lifecycle management at runtime. Furthermore, it is also responsible for instantiating both VMs and virtual links for the running services inside the resource domains.

\section{Resource Marketplace}

The resource marketplace implements a dynamic resource discovery approach, initiated by the NECOS slice provider. The design was influenced by existing online marketplaces for flight pricing and hotel reservations, which provided a working operational model to follow. Rather than configuring predetermined providers sets in a federation approach, NECOS applies a more flexible run-time model, where an auctioneer component announces a call-for-proposals for resource offers on a slice part, aggregating alternative offers by bidders. The aggregator can be a sub-system of any resource provider participating in NECOS, or in a more complex setting, it can be an external aggregator that provides either compound offers or offers at a price point. The results of this auction process are collected and communicated back to the slice provider, who selects the actual slice resources.

The primary reason for these design choices was to provide a mechanism that reaches and interacts with resource providers in multi-geographic places in order to provision end-to-end cloud-network slices. Existing federation approaches often lack coverage to these network kinds. The second reason is that, in general, slices are created in a more dynamic fashion than federation agreements, therefore, a highly-dynamic, market-inspired provider searching mechanism is required. In contrast to delegating resource management to the respective domains, our approach trades a potential performance increase at the domain-level resource adaptations for a greater flexibility, since it permits changes in the slice graph, e.g., allocating services to new providers, service reallocation to other slice parts, etc. A detailed description of the marketplace can be found in [8].

\section{Resource Providers}

Resource providers are those organisations in charge of providing the set of required resources to provision slice parts. Other types of resources can be manifested by organisations featuring MEC, networked sensors, or wireless networks. The allocation of a new VIM (for DC slice parts), or a new WIM (for network slice parts) is also the responsibility of the resource provider, allowing the slice resource orchestrator to remotely manage and utilise the resources.

\section{E. Business Case}

NECOS relies on the Slice-as-a-Service model to set up network-cloud slices for a wide-range of business oriented applications [14]. Here, we briefly present a business case built around the NECOS platform, by aligning with the business model CANVAS approach, for creating slices which utilise a logistics IoT scenario:

Value proposition. The business case relates to the provisioning of network-cloud slices accommodating services for international logistic companies, allowing tracking of assets during 
worldwide delivery, with the customer being interested in the geographical location, temperature, humidity, etc.

Customer segments. The end-customer acting as a NECOS tenant, can directly be any of international logistics, delivery, or transportation companies, or can be providers of Internetbased services to the logistics companies.

Key resources. The business-case requires NECOS platforms deployed in a number of geographical locations, owned by unique or different organizations. These can be network operators, cloud providers, or new business entities, acting as NECOS slice, resource marketplace, cloud, network, or IoT infrastructure providers.

Key activities. The key activities concern the creation, activation, efficient run-time operation and teardown of cloudnetwork slice instances and service elements, tailored to the evolution of the customers needs.

Key partnerships. Slice providers receiving slice creation requests may transfer part of the requests to one or more partners, in case of resource-shortage. A novelty of the NECOS approach enables partnerships of existing or new types of business entities, such as sensor network providers, participating in NECOS roles.

Revenue and cost streams. Both revenue from and cost of a slice can be based on cost models being proportional to the amount of DC and network resources needed, the utilization of the sensor network, and the slice duration.

\section{NECOS FUNCTIONAL BLOCKS}

We now describe the NECOS architecture functional blocks which are presented in Figure 2 (with more details in [11]).

Service Orchestrator: Residing in the tenant's domain, this component is responsible for deploying and managing tenant services on the allocated slice, by interacting with the slice resource orchestrator.

Service Activator: This component is responsible for 2 tasks: (i) passing the tenant's Slice Description to the NECOS slice provider, and (ii) handling the response, which informs the tenant's service orchestrator about the slice status.

Slice Specification Processor: This component transforms the tenant's slice descriptor, which only requires a service specification, into an information-rich slice creation request for the slice builder, which includes resource options, geographical info, decomposition for slice parts, etc.

Slice Builder: This component builds a full end-to-end cloudnetwork slice from a set of constituent slice parts. It is responsible for selecting, among the alternative offers, each slice part that the slice broker reports back as output from the discovery process via the resource marketplace.

Slice Resource Orchestrator (SRO): This component joins together the slice parts into a single aggregated slice to be used for service deployment, orchestration, and run-time lifecycle management, including the placement and embedding, into the resource domains, of the VMs and virtual links for the services.

Infrastructure \& Monitoring Abstraction (IMA): In order to interact with various VIM/WIM instances and the monitoring subsystems, the NECOS slice provider relies on IMA mechanisms which abstract away technology specific details through plug-in API adaptors. The IMA subsystem provisions the tenant services and also monitors the underlying resources, collecting additional telemetry data that is not available via the service-level monitoring interface.

DC Slice Controller: This component creates Data Center slices so that a given slice part is configured with compute and storage resources, as well as returning a handle to the respective VIM manager. This VIM can either be an instance deployed on-demand, complying with the tenant's specification, or a existing running VIM in that resource domain, offering an isolated interaction point for the tenant.

WAN Slice Controller: For each network domain, a WAN slice controller instantiates each new network slice to interconnect two DC slices. Like the VIM, instances of the WIM can be deployed on-demand, per tenant, or shared through an existing WIM using an isolation shim.

Slice Broker: This component manages the resource discovery process of the marketplace by decomposing a full slice request into a per slice part call-for-proposals. This triggers a bidding process involving the slice agents in each provider domain. The broker then aggregates the alternative responses, each annotated with a set of costs, and sends them to the slice builder.

Slice Agent: Running in each DC/WAN provider domain, this component's role is to receive slice part requests and to match the detailed slice part requirements to available resources reported by its own DC/WAN controller. If a callfor-proposals match was successful, it then returns an offer for the slice part.

\section{EXPERIMENTAL EVALUATION}

In order to validate whether the NECOS LSDC platform is able to support the list of novel features presented in Section II. different software components were developed and evaluated in a set of experiments. These experiments are grouped into three categories according to their functional domain and capabilities: (i) marketplace-based resource discovery; (ii) intelligent vertical elasticity functionalities; and (iii) life-cycle management of end-to-end slices and services. Additional experiments are provided through demonstrators including on end-to-end slicing for both logistics IoT and touristic CDN services.

Although the prototype artifacts were not engineered for performance but rather for a proof-of-concept demonstration of the main NECOS novelties, when using lightweight components as in [7], the processing steps, from sending a slice request to having a handle returned to an executing manager, take around six seconds, depending on the capacity and load of the server.

The experiments were executed on a geographically distributed testbed deployed on 6 sites: 4 located in Brazil (Pará, Goiás, Campinas, and São Carlos), and 2 in Europe (Greece and Spain). In addition, 14 resource providers were emulated

\footnotetext{
${ }^{1}$ Pointers to videos, source code and reproducibility instructions of all NECOS demonstrators are available at: http://www.h2020necos.eu/demonstrators/
} 
TABLE I

Performance Results of the MarketPlace

\begin{tabular}{|lllll|}
\hline $\begin{array}{l}\text { Slice Request } \\
\text { 3-tuples }\end{array}$ & $\begin{array}{l}\text { Alternative } \\
\text { DC Parts }\end{array}$ & $\begin{array}{l}\text { Alternative } \\
\text { Net Parts }\end{array}$ & $\begin{array}{l}\text { Slice } \\
\text { Alternatives }\end{array}$ & $\begin{array}{l}\text { Wall Time } \\
(\mathrm{sec})\end{array}$ \\
\hline \hline$(2,1,4)$ & 11 & 27 & 27 & 11.3 \\
\hline$(3,2,6)$ & 14 & 40 & 320 & 18.8 \\
\hline$(4,5,8)$ & 18 & 256 & 26,924 & 91.4 \\
\hline$(6,7,24)$ & 55 & 1,755 & 50,000 & 605.8 \\
\hline$(8,9,32)$ & 73 & 2,205 & 50,000 & 763.1 \\
\hline
\end{tabular}

and used during the tests to better assess the experimental behavior on a larger scale. RAN-level slicing is out of the experiments since the testbed lacks wireless domains with virtualization and softwarization substrates.

\section{A. Marketplace}

The novelties of the NECOS platform with respect to the Slice-as-a-Service model F1, the lightweight architecture F2, the multi-domain operation F3, and the Marketplace's dynamic discovery of distributed resources F6, were evaluated in a usecase involving up to 20 DC resource providers and 3 network resource providers. A slice request generator was used to introduce Tenant slice request workloads.

The size of each slice request is characterised by a 3-tuple, indicating the different number of DC slice parts, network slice parts, and virtual functions hosted, presented respectively as $(d c, n e t, v f)$ in column 1 of Table II The required attributes of the complete slice request [8] were populated with random

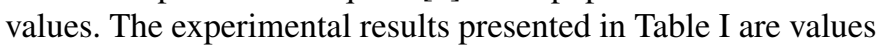
for each slice request, averaged over five runs. Columns 2 and 3 present the number of DC and Network Slice Part alternatives collected, column 4 has the alternatives of endto-end slices considered (with a max value of 50,000), and, finally, column 5 the average time since the initial request to make the final slice selection. From these results and similar experiments, we conclude that the Marketplace is (i) fully functional and (ii) scales well for a wide range of parameters, including the number of slice parts, virtual functions, attributes' range, and diversity of resource providers.

\section{B. Vertical elasticity enabled by Machine Learning}

One of the core functions of the NECOS Slice Resource Orchestrator (SRO) is the run-time management of the slice life-cycle, which includes the capability to carry out dynamic upgrades and downgrades of the resources allocated to the slice, to respond to demand changes. This capability, which is called elasticity [12], consists of two models: vertical elasticity entailing the change of the amount of resources of the same slice parts; horizontal elasticity referring to the change of resources through the addition or removal of slice parts. This experiment summarizes the tests conducted to confer vertical elasticity to the SRO, by leveraging machine learning.

Although this experiment also supports the validation of NECOS novel features F1 and F2, it is specifically focused on demonstrating feature F4, the intelligent elasticity, through an SRO implementing a machine learning algorithm that receives

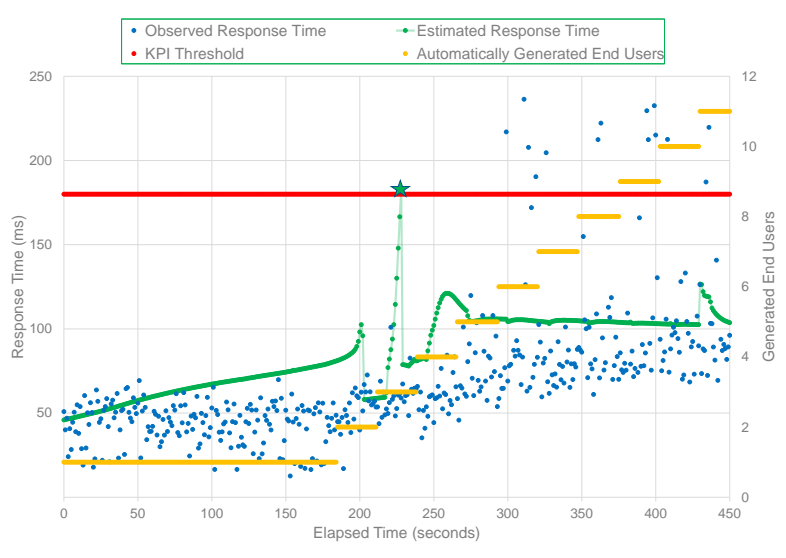

Fig. 3. Estimated Response Time versus Observed Response Time as a function of the number of end users consuming the Cassandra service

slice KPI metrics, and infers KPIs associated to the slice service level agreement (SLA) metrics. Through the time evolution of these KPIs, it is possible to predict the need to trigger upscale or downscale vertical elasticity processes to avoid SLA violations, while providing resource efficiency.

The experiment consists of a service using the Cassandra database, with the KPI of interest being the read-write time operations. The service is deployed on a slice over the distributed testbed and is characterized by infrastructure parameters like $\mathrm{CPU}$, memory, network traffic, and others. As the number of service users accessing the service increases or decreases (via a load generator), the monitored resources are affected and the read-write time service KPIs are impacted.

The SLA metric defined with a threshold of "response time below 180 milliseconds" means that whenever the orchestrator predicts an SLA violation in the next 30 seconds, the elasticity process is started. The timings collected for a vertical elasticity process are plotted in Figure 3. At around 225 seconds after the start of the time window, the orchestrator foresees an SLA violation (green star) and proactively triggers the vertical elasticity operations to modify the slice with additional resources. The elasticity process takes a couple of seconds and this fixes the foreseen violation, as can be seen by the response times that are mainly maintained under the specified SLA, even as the number of users keeps growing.

\section{Full slice lifecycle in multi-site distributed environment}

We evaluate the full process of end-to-end, multi-domain Slice-as-a-Service delivery through the deployment of a touristic CDN service on a slice that includes resource domains in Brazil and Europe (see Figure 4). This experiment uses all the components of the NECOS architecture of Figure 2, and is devised to validate a rich set of the novel features of NECOS.

The touristic CDN service, highlighted via a blue dottedline in Figure 4 , operates on top of a cloud-network slice and delivers touristic content to users based on their geographic location. For the sake of simplicity, the figure only shows those providers that met the requirements of the requested slice, i.e., resource providers along their slice controller and slice agent instances located in Campinas, São Carlos, Spain, and Greece. 


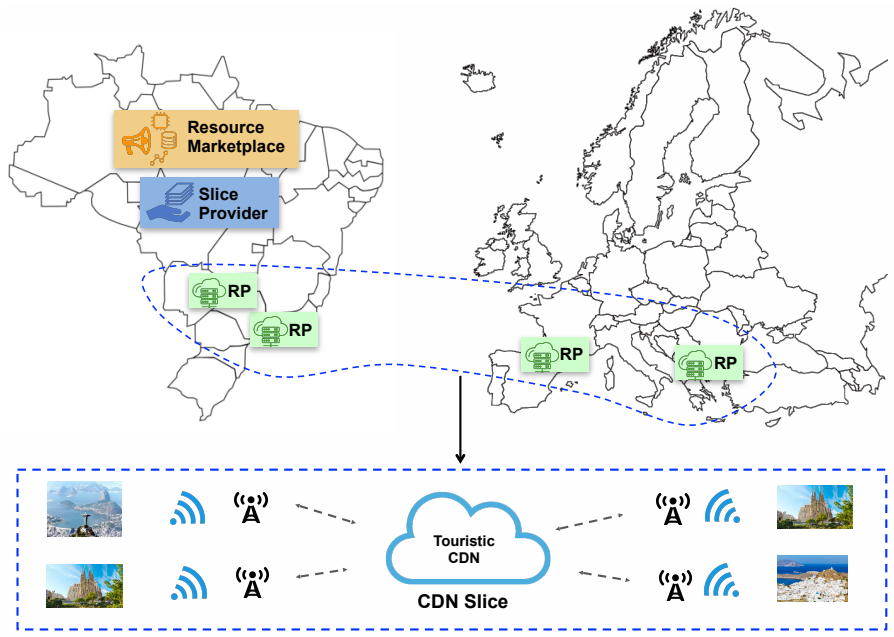

Fig. 4. Touristic CDN service slice deployment in resource provider facilities in Brazil and Europe.

The process workflow is executed multiple times and, for each run, data is sampled to gather the times to create the slice, deploy the service, and decommissioning the slice.

In Figure 5, we can observe that, on average, the process workflow takes almost 10 minutes $(\approx 578$ s) to create the slice, and nearly 6 minutes $(\approx 403 \mathrm{~s}$ ) to deploy the touristic CDN service, resulting in a total of 16 minutes to fully provision the slice with its service. We now look into the duration of the different operations, namely, the time spent looking for candidate slice parts, both DC and network, discovering the slice parts (SPD), instantiating the DC slice parts (DC $\mathrm{SPI}$ ) in the selected resource providers, and setting up the network slice parts to form the end-to-end cloud network slice (Net SPI). In our experiments, it takes on average less than 2 minutes to find 7 slice parts (4 DC slice parts and 3 Net slice parts) among all the combinations involving 20 resource providers distributed across Europe, Brazil (SPD in Figure 5p and the 14 emulated resource providers. On average, it takes less than 9 minutes to instantiate a DC slice part (DC SPI segment of slice creation) containing a fully-configured Kubernetes cluster and only less than 2 seconds to setup a VxLAN network slice part for the touristic CDN slice (Net SPI fraction of slice creation).

These times should be viewed with a perspective of our prototype establishing a trans-oceanic slice - an entity that may last for days, weeks or even years - atop the physical and virtual resources of the testbed infrastructure. Therefore, these numbers should be considered as acceptable overheads for a relatively long lasting slice.

\section{CONClusions AND OUtLOOK}

The NECOS cloud-network slicing concept presents a set of innovative characteristics that allows for slicing using the a Slice-as-a-Service model, in which service components are dynamically mapped into an end-to-end slice based on resources from different providers. The NECOS platform offers participating resource providers the ability to federate their infrastructures through a marketplace approach. This enables

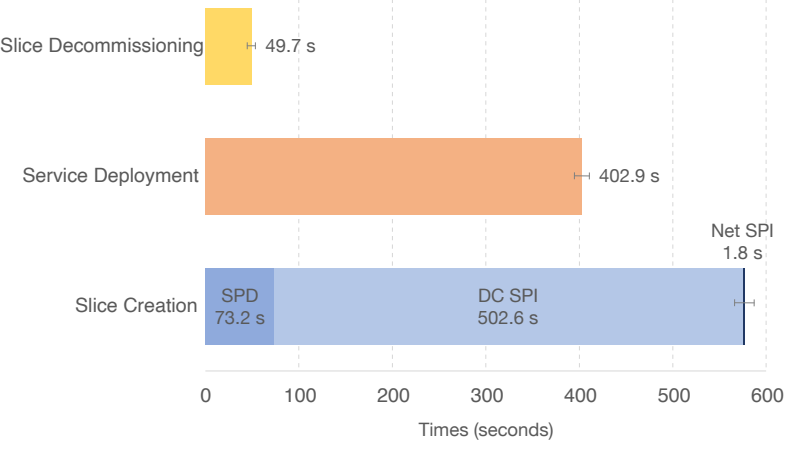

Fig. 5. Slice lifecycle elapsed times of the touristic CDN trial.

the execution of tenant services using virtualized resources to span across the cloud/network infrastructures offered by providers presenting different configurations. Such a concept is not purely technical, as it also considers business, cultural, and geographical relationships among domains. A number of experiments over instances of the NECOS architecture have served to prove its core concepts and novelties.

There are still many open challenges around the broader vision of slicing, for example: (i) full slice isolation, i.e., highly efficient creation of isolation-guaranteed slices for each of the data, control, management, and service-planes, which demands the development of enablers for safe, secure, and efficient slice multi-tenancy; and (ii) slicing service mapping, i.e., setting an efficient service mapping model bound over network cloud slicing, specifying policies and methods to honour service requirements without imposing infrastructure re-engineering. We expect the evolution of slicing to keep embracing heterogeneous resources at all $\mathrm{HW} / \mathrm{SW}$ layers in order to realize service-tailored end-to-end architectures. Using innovative business and operational models, governed by the economies of sharing, where multi-tenancy at multiple realms becomes the norm, slicing will take the networking principle of resource multiplexing to new levels.

\section{ACKNOWLEDGMENT}

Work supported by the H2020 EU-Brazil collaborative call, grant no. 777067 (NECOS), funded by the European Commission and the Brazilian Ministry of Science, Technology, Innovation, and Communication (MCTIC) through CTIC/RNP.

\section{REFERENCES}

[1] A. A. Barakabitze, A. Ahmad, R. Mijumbi, and A. Hines, "5G network slicing using SDN and NFV: A survey of taxonomy, architectures and future challenges," Computer Networks, vol. 167, p. 106984, 2020.

[2] L. U. Khan, I. Yaqoob, N. H. Tran, Z. Han, and C. S. Hong, "Network slicing: Recent advances, taxonomy, requirements, and open research challenges," IEEE Access, vol. 8, pp. 36009-36028, 2020.

[3] A. Galis, F. Tusa, S. Clayman, C. Rothenberg, and J. Serrat, "Slicing 5G Networks: An Architectural Survey," in Wiley 5G Ref: The Essential 5G Reference Online. John Wiley \& Sons, Inc, 2020, pp. 1-41. [Online]. Available: https://onlinelibrary.wiley.com/doi/abs/ 10.1002/9781119471509.w5GRef095

[4] T. Taleb, I. Afolabi, K. Samdanis, and F. Z. Yousaf, "On multidomain network slicing orchestration architecture and federated resource control," IEEE Network, vol. 33, no. 5, pp. 242-252, 2019. 
[5] NECOS, "EU-Brazil - Novel Enablers for Cloud Slicing," 2017, http: //www.h2020-necos.eu (Last Accessed: Nov 2020).

[6] S. Clayman, F. Tusa, and A. Galis, "Extending Slices into Data Centers: the VIM on-demand model," in IEEE 9th International Conference on Network of the Future - NoF, Poznań, Poland, 19-21 November 2018.

[7] S. Clayman, F. Tusa, A. Galis, and L. Contreras, "WIM on-demand A modular approach for managing network slices," in IEEE Conference on Network Softwarization - Netsoft, Ghent, 29 June-3 July 2020.

[8] P. D. Maciel, F. L. Verdi, P. Valsamas et al., "A Marketplace-based Approach to Cloud Network Slice Composition Across Multiple Domains," in 2nd Workshop on Advances in Slicing for Softwarized Infrastructures (S4SI), co-hosted at the 5th IEEE NetSoft, Paris, 24-28 June 2019.

[9] F. S. D. Silva, M. O. Lemos, A. Medeiros, A. V. Neto, R. Pasquini, D. Moura, C. Rothenberg, L. Mamatas, S. L. Correa, K. V. Cardoso et al., "NECOS project: Towards lightweight slicing of cloud federated infrastructures," in 4th IEEE Conference on Network Softwarization and Workshops (NetSoft). IEEE, 25-29 June 2018.

[10] P. Valsamas, P. Papadimitriou, I. Sakellariou, S. Petridou, L. Mamatas, S. Clayman, F. Tusa, and A. Galis, "Multi-PoP Network Slice Deployment: A Feasibility Study," in IEEE CloudNet, 4-6 November 2019.

[11] NECOS project, "D3.2: NECOS System Architecture and Platform Specification. V2," Tech. Rep., April 2019, (Last Accessed: Nov 2020). [Online]. Available: http://www.maps.upc.edu/public/necos_d3.2.v4.11_ final_web.pdf

[12] NECOS Project, "D5.2: Intelligent Management and Orchestration," Tech. Rep., October 2019, (Last Accessed: Nov 2020). [Online]. Available: http://www.maps.upc.edu/public/D5.2\%20final.pdf

[13] ETSI, "Zero-touch network and Service Management (ZSM) Industry Specification Group (ISG) ," 2019, https://www.etsi.org/committee/zsm (Last Accessed: Nov 2020).

[14] NECOS project, "D2.2: Consolidated definition of use cases, business models and requirements analysis," Tech. Rep., December 2018, (Last Accessed: Jan 2021). [Online]. Available: http://www.maps.upc.edu/ public/d2.2_final_v2.0.pdf

Dr. Stuart Clayman is a Principal Research Fellow at the Electronic Engineering Department, University College London, UK.

Dr. Augusto Venâncio Neto is an Associate Professor at the Informatics and Applied Mathematics Department, Federal University of Rio Grande do Norte, Brazil

Dr. Fábio L. Verdi is an Associate Professor at the Computing Department, Federal University of São Carlos, Brazil.

Dr. Ilias Sakellariou is an Assistant Professor at the Department of Applied Informatics, University of Macedonia, Greece.

Dr. Silvio Sampaio is an Assistant Professor at the Digital Metropolis Institute, Federal University of Rio Grande do Norte, Brazil.

Dr. Sand Correa is an Associate Professor at the Instituto de Informática, Universidade Federal de Goiás, Brazil.

Dr. Lefteris Mamatas is an Assistant Professor at the Department of Applied Informatics, University of Macedonia, Greece.

Dr. Rafael Pasquini is an Associate Professor, in the Faculty of Computing at Universidade Federal de Uberlândia (UFU), Brazil.

Dr. Kleber Cardoso is an Associate Professor at the Instituto de Informática Universidade Federal de Goiás, Brazil.

Dr. Francesco Tusa is a Research Associate at the Electric and Electronic Engineering Department, University College London, UK.

Dr. Christian Rothenberg is an Assistant Professor at the University of Campinas, Brazil.

Dr. Joan Serrat is a Full Professor at Universitat Politècnica de Catalunya, Spain. 